\title{
An extended-G geometric family
}

\author{
Gauss M. Cordeiro ${ }^{1}$, Giovana O. Silva ${ }^{2 *}$ and Edwin M. M. Ortega ${ }^{3}$
}

\author{
*Correspondence: giovana@ufba.br \\ 2 Departamento de Estatística, \\ Universidade Federal da Bahia, Av. \\ Adhemar de Barros, s/n, 40170-110 \\ Salvador, Brazil \\ Full list of author information is \\ available at the end of the article
}

\begin{abstract}
We introduce and study the extended-G geometric family of distributions, which contains as special models some important distributions such as the XTG (Xie et al. 2002) geometric, Weibull geometric, Chen (Chen 2000) geometric, Gompertz geometric, among others. This family not only includes distributions with bathtub and unimodal failure rate functions but provides a broader class of monotone failure rates. Its density function can be expressed as a linear mixture of extended-G densities. We derive explicit expansions for the ordinary and incomplete moments, generating function, mean deviations and Rénvy entropy. The density of the order statistics can also be given as a linear mixture of extended-G densities. The model parameters are estimated by maximum likelihood. The potentiality of the new family is illustrated by means of an application to real data.
\end{abstract}

MSC: 60E05, 62P10, 62P30

Keywords: Expected information, Extended-G distribution, Geometric distribution, Lifetime distribution, Maximum likelihood estimation

\section{是 Springer}

\section{Introduction}

In the last few years, new classes of distributions were proposed by extending the Weibull distribution to cope with bathtub shaped failure rates. A good review of some of these models is addressed by Pham and Lai (2007). Among these models, we point out the exponentiated Weibull (Mudholkar et al. 1995, 1996), additive Weibull (AW) (Xie and Lai 1995), XTG (Xie et al. 2002), modified Weibull (MW) (Lai et al. 2003), beta exponential (Nadarajah and Kotz 2006), BLZ (Bebbington et al. 2007), generalized modified Weibull (GMW) (Carrasco et al. 2008), beta modified Weibull (BMW) (Silva et al. 2010a) and beta Weibull geometric (Cordeiro et al. 2013) distributions.

Alternatively, various works introduced more flexible distributions in modeling monotone or unimodal failure rates but it is not useful for modeling the bathtub shaped failure rates. Adamidis and Loukas (1998) defined the exponential geometric distribution to model lifetime data with decreasing hazard rate function (hrf). Gupta and Kundu 1999, 2001a, b proposed the generalized exponential (GE) (also called the exponentiated exponential) distribution, and investigated some of its mathematical properties. This distribution has only increasing or decreasing hrf. Following the same idea of the GE distribution, Silva et al. (2010b) proposed the generalized exponential geometric (GEG) model and demonstrated that its hrf can be increasing, decreasing or unimodal. Another generalization of the Weibull for modeling monotone or unimodal failure rates, referred to as

C 2016 Cordeiro et al. Open Access This article is distributed under the terms of the Creative Commons Attribution 4.0 International License (http://creativecommons.org/licenses/by/4.0/), which permits unrestricted use, distribution, and reproduction in any medium, provided you give appropriate credit to the original author(s) and the source, provide a link to the Creative Commons license, and indicate if changes were made. 
the Weibull geometric (WG) distribution, was investigated by Barreto-Souza et al. (2011). The Kumaraswamy log-logistic distribution was studied by de Santana et al. (2012). This generalization contains distributions with unimodal and bathtub shaped hrfs.

In this paper, we propose the new extended-G geometric (EGG) family of lifetime models by compounding the extended-G (EG) (Nadarajah and Kotz 2005) class of models and the geometric distribution. This family has two important aspects: it involves one additional shape parameter to the baseline model and the new parameter has a clear physical interpretation. We expect that it will attract wider applications in biology, medicine and reliability, and other areas of research.

Further, several distributions are obtained as special cases of this family including the extended exponential geometric (EEG), modified Weibull geometric (MWG), exponential power geometric (EPG), log-Weibull geometric (LWG), generalized power Weibull geometric (GPWG), among several others. Besides these distributions, the EGG family contains other promising new distributions as, for example, the additive Weibull geometric (AWG) and XTG geometric (XTGG) distributions (see "Appendix"). Due to its flexibility in accommodating different forms of the risk function, the new family is an important tool to be used in a variety of problems in modeling survival data. Various EGG distributions are not only convenient for modeling comfortable bathtub-shaped failure rates but they are also suitable for testing goodness-of-fit of some special models.

The paper is organized as follows. In Section 2, we define the EGG family and demonstrate that its probability density function (pdf) is given as a linear mixture of EG densities. In Sections 3 and 4, we derive the moments and moment generating function (mgf), respectively. The mean deviations and Bonferroni and Lorenz curves are obtained in Section 5. In Section 6, we demonstrate that the Rényi entropy of the EGG family is a linear combination of EG entropies with different scale parameters. In Section 7, the density function of the EGG order statistics is expressed as a linear mixture of EG densities. Maximum likelihood estimation of the model parameters is addressed in Section 8. In Section 9, we give an application to ozone level data to prove empirically the potentiality of the proposed family. Concluding remarks are provided in Section 10. Some special models are presented in the "Appendix".

\section{The EGG family}

The cumulative distribution function (cdf) of the EG class is defined by

$$
G_{\alpha, \tau}(y)=1-\exp \{-\alpha H(y)\}, y>0
$$

where $\alpha>0$ is a rate parameter, $H(y)$ is a monotonically increasing function of $y$ with the only limitation $H(y) \geq 0$ and $\tau$ denotes the vector of unknown parameters in $H(y)$. If $H(y)$ is a power function, Eq. (1) reduces to the Weibull distribution. The EG class pdf is given by

$$
g_{\alpha, \tau}(y)=\alpha h(y) \exp \{-\alpha H(y)\}, y>0
$$

where $h(y)=\partial H(y) / \partial y$. We denote by $Y_{\alpha} \sim E G(\alpha, \tau)$ a random variable $Y_{\alpha}$ having density function (2). The MW distribution is a special case of (2) when $H(y)=y^{\gamma} \exp (\lambda y)$, where $\gamma>0$ and $\lambda \geq 0$. Clearly, the Weibull distribution is obtained as a basic exemplar when $\lambda=0$. 
The EG quantile function (qf) is given by

$$
Q_{\alpha, \tau}(u)=H^{-1}\left\{-\frac{1}{\alpha} \log (1-u)\right\},
$$

which plays an important role in the algebraic developments in the paper. We only need the inverse function of $H(y)$ to determine (3). Table 1 lists closed-form inverses of $H(\cdot)$ for some EG sub-models.

Let $Z$ be a geometric random variable with probability mass function given by $P(z ; p)=$ $(1-p) p^{z-1}$ for $z \in N$ and $p \in(0,1)$. Suppose that $\left\{Y_{i}\right\}_{i=1}^{Z}$ are independent and identically distributed (iid) random variables having the same density function (2), where the unknown number $Z$ of random variables follows this geometric distribution. We assume that the random variables $Z$ and $Y_{i}^{\prime}$ s are independent. The EGG family is defined by $X=\min \left(\left\{Y_{i}\right\}_{i=1}^{Z}\right)$, where $Z$ has this geometric distribution. We provide three interesting situations for applying this family:

- Reliability - the new distribution can arise in series and parallel systems with identical components, which appear in many industrial applications and biological organisms;

- Times to the first and last failure - suppose the failure of a device occurs due to the presence of an unknown number $Z$ of initial defects of same kind, which can be identifiable only after causing failure and are repaired perfectly. Let $Y_{i}$ be the time to the failure of the device due to the $i$ th defect, for $i \geq 1$. Under the assumptions that the $Y_{i}$ 's are iid EG random variables independent of $Z$, the EGG distribution is appropriate for modeling the times to the first and last failures, respectively;

- Time to relapse of cancer under the first-activation scheme - suppose that an individual in the population is susceptible to a certain type of cancer. Let $Z$ be the number of carcinogenic cells for that individual left active after the initial treatment and denote by $Y_{i}$ the time spent for the $i$ th carcinogenic cell to produce a detectable cancer mass, for $i \geq 1$. Under the assumptions that $\left\{Y_{i}\right\}_{i \geq 1}$ is a sequence of iid EG random variables independent of $Z$, we conclude that the time to relapse of cancer of a susceptible individual has the EGG distribution.

The conditional density function of $X$ given $Z=z$ is given by

$$
f(x \mid z ; \alpha, \boldsymbol{\tau})=z \alpha h(x) \exp \{-\alpha H(x)\}[1-\exp \{-\alpha H(x)\}]
$$

Table 1 Inverse function $x=H^{-1}(y)$ for some EG models

\begin{tabular}{ll}
\hline Distribution & $x=H^{-1}(y)$ \\
\hline Exponential power & $\frac{[\log (y+1)]^{1 / \beta}}{\lambda}$ \\
Chen & {$[\log (y+1)]^{1 / \beta}$} \\
XTG & $\lambda[\log (y / \lambda+1)]^{\frac{1}{\beta}}$ \\
Log-Weibull & $\sigma \log (y)+\mu$ \\
Kies & $\frac{y^{1 / \beta} \sigma+\mu}{y^{1 / \beta}+1}$ \\
Generalized power Weibull & $\beta\left[(y+1)^{1 / \theta}-1\right]^{1 / \alpha_{1}}$ \\
BLZ & $\frac{\log (t) \pm \sqrt{[\log (y)]^{2}+4 \alpha_{1} \beta}}{2 \alpha_{1}}$ \\
Gompertz & $\frac{\log \left(\alpha_{1} y+1\right)}{\alpha l_{1}}$ \\
Pham & {$\left[\frac{\log (1+y)}{\log \left(a_{1}\right)}\right]^{1 / \alpha_{1}}$} \\
\hline
\end{tabular}


and then the EGG density function reduces to

$$
f(x ; p, \alpha, \boldsymbol{\tau})=\alpha(1-p) h(x) \exp \{-\alpha H(x)\}[1-p \exp \{-\alpha H(x)\}]^{-2}, \quad x>0 .
$$

The MWG distribution is obtained from (4) when $H(x)=x^{\gamma} \exp (\lambda x)$, where $\gamma>0$ and $\lambda \geq 0$. Further, the WG distribution is also obtained as a special case when $\lambda=0$. The EG distribution follows as the limiting distribution (the limit is defined in terms of the convergence in distribution) of the EGG distribution when $p \rightarrow 0^{+}$. On the other hand, if $p \rightarrow 1^{-}$, we obtain the distribution of a random variable $Y$ such that $P(Y=0)=1$.

Hereafter, a random variable $X$ having density function (4) is denoted by $X \sim$ $E G G(p, \alpha, \tau)$. The EGG cdf is given by

$$
F(x ; p, \alpha, \boldsymbol{\tau})=\frac{1-\exp \{-\alpha H(x)\}}{1-p \exp \{-\alpha H(x)\}}, \quad x>0 .
$$

The hrf corresponding to (4) becomes

$$
h(x ; p, \alpha, \tau)=\frac{\alpha h(x)}{1-p \exp \{-\alpha H(x)\}}, \quad x>0 .
$$

The EGG family contains as special models some well-known distributions. Several new models can also be easily generated as those listed in the "Appendix". Plots of the density functions (24)-(25) (given in the "Appendix") and the corresponding hrfs for the XTGG and MWG distributions are displayed in Figs. 1 and 2, respectively.

For $|z|<1$ and $\rho>0$, we can write

$$
(1-z)^{-\rho}=\sum_{j=0}^{\infty} \frac{\Gamma(\rho+j)}{\Gamma(\rho) j !} z^{j} .
$$

A useful representation for the EGG pdf $(x>0)$ follows by applying (7) in Eq. (4)

$$
f(x ; p, \alpha, \boldsymbol{\tau})=\sum_{j=0}^{\infty} w_{j} g_{(j+1) \alpha, \tau}(x),
$$

where $w_{j}=(1-p) p^{j}$ for $j=0,1, \ldots$ and $g_{(j+1) \alpha, \tau}(x)$ denotes the density function of the random variable $Y_{(j+1) \alpha}$. Evidently, $\sum_{j=0}^{\infty} w_{j}=1$. Note that the right-hand side of (8) is mathematically more tractable than the density of $X$. So, the EGG density function can be expressed as a linear mixture of EG densities. Equation (8) is very useful to derive

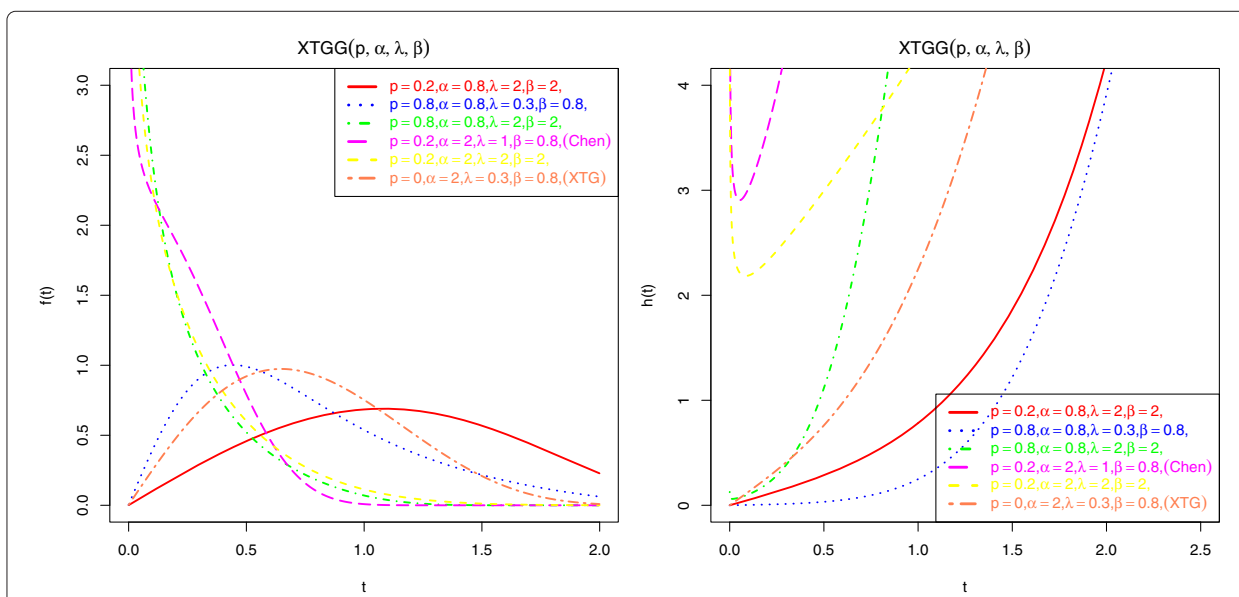

Fig. 1 Plots of the XTGG density and hazard rate functions for some parameter values 

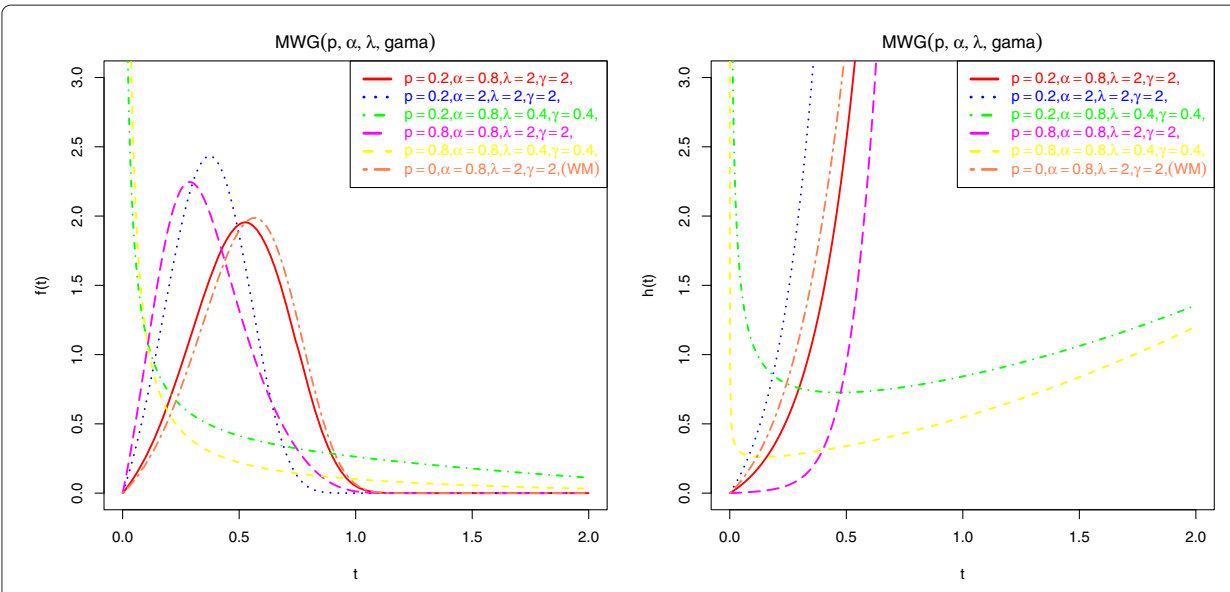

Fig. 2 Plots of the MWG density and hazard rate functions for some parameter values

some mathematical quantities (for example, ordinary, incomplete and factorial moments and mgf) for the EGG family from an infinite weighted linear combination of those EG quantities.

\section{Moments}

In this section, we derive an explicit expression for the EGG moments. Let $\beta_{s}(\alpha, \tau)=$ $E\left(Y_{\alpha}^{s}\right)$ be the $s$ th moment of the EG class (2) with parameters $\alpha$ and $\boldsymbol{\tau}$. The $s$ th moment of $X$, say $\mu_{s}^{\prime}$, can be expressed in terms of the corresponding moments of EG distributions. In fact, we obtain a very simple expression from (8)

$$
\mu_{s}^{\prime}=E\left(X^{s}\right)=\sum_{j=0}^{\infty} w_{j} \beta_{s}((j+1) \alpha, \boldsymbol{\tau}) .
$$

Equation (9) reveals that the moments of $X$ are linear combinations of the corresponding moments of $Y_{(j+1) \alpha}$ for $j \geq 0$. So, the moments of the EGG family are expressed as infinite linear combinations of those EG moments.

Next, we provide three alternative expressions for $\beta_{s}(\alpha, \tau)$. First, by substituting $u=$ $Q_{\alpha, \tau}(y)$ given by (3) in the sth moment of $Y_{\alpha}$ determined from (2), we obtain

$$
\beta_{s}(\alpha, \tau)=\int_{0}^{1} Q_{\alpha, \tau}(u)^{s} d u
$$

A second formula follows from (2) by changing variable $v=H(y)$

$$
\beta_{s}(\alpha, \tau)=s \int_{0}^{\infty} p_{s, \tau}(v) \mathrm{e}^{-\alpha \mathrm{v}} \mathrm{dv}=\mathrm{P}_{\mathrm{s}, \boldsymbol{\tau}}(-\alpha),
$$

where $p_{s, \tau}(v)=\frac{H^{-1}(\nu)^{s-1}}{h\left(H^{-1}(v)\right)}$ and $P_{s, \tau}(-\alpha)=\mathfrak{L}\left(p_{s, \tau}(v) ; \alpha\right)$ is the Laplace transform of $p_{s, \tau}(v)$. A third formula for $\beta_{s}(\alpha, \tau)$ comes from the Mellin transform of $h(y) \mathrm{e}^{-\alpha \mathrm{H}(\mathrm{y})}$, namely

$$
\beta_{s}(\alpha, \tau)=\alpha \mathrm{M}\left(\mathrm{h}(\mathrm{y}) \mathrm{e}^{-\alpha \mathrm{H}(\mathrm{y})} ; \mathrm{s}+1\right) .
$$

Several tables provide Mellin transforms for common functions. The Laplace and Mellin transforms are defined in Prudnikov et al. (1986). Equations (9)-(11) are the main results of this section. They can be used to obtain analytically or numerically the moments of several EGG special models. 
Further, we provide an application of (9) in conjunction with (10) and (11). Substituting $H(y)$ of the XTG distribution given in the "Appendix" into Eq. (11) and assuming that $m=s / \beta$ is an integer, and after some algebra, we have

$$
\begin{aligned}
\beta_{s}(\alpha, \beta, \lambda) & =s \int_{0}^{\infty} v^{s-1} \exp (\lambda \alpha) \exp \left\{-\lambda \alpha \exp \left[(v / \lambda)^{\beta}\right]\right\} d v \\
& =\left.m \lambda^{s} \exp (\lambda \alpha) \frac{\partial^{m-1}(\lambda \alpha)^{-t} \Gamma(t, \lambda \alpha)}{\partial t^{m-1}}\right|_{t=0},
\end{aligned}
$$

where $\Gamma(t, \alpha)=\int_{t}^{\infty} w^{\alpha-1} \mathrm{e}^{-\mathrm{w}} \mathrm{dw}$ is the upper incomplete gamma function. Equation (12) gives the moments of the XTG distribution. Hence, we can express the XTGG moments by combining Eqs. (9) and (12).

The central moments $\left(\mu_{s}\right)$ and cumulants $\left(\kappa_{s}\right)$ of $X$ can be obtained from (9) as

$$
\mu_{s}=\sum_{j=0}^{s}(-1)^{j}\left(\begin{array}{l}
s \\
j
\end{array}\right) \mu_{1}^{\prime s} \mu_{s-j}^{\prime} \quad \text { and } \kappa_{s}=\mu_{s}^{\prime}-\sum_{j=1}^{s-1}\left(\begin{array}{c}
s-1 \\
j-1
\end{array}\right) \kappa_{j} \mu_{s-j}^{\prime},
$$

respectively, where $\kappa_{1}=\mu_{1}^{\prime}$. The skewness and kurtosis measures can be determined from the central moments using well-known relationships. Plots of the skewness and kurtosis for some parameter values as functions of $\alpha$ for the XTGG and MWG distributions are displayed in Figs. 3 and 4, respectively.

Finally, for empirical purposes, the shape of many distributions can be usefully described by the incomplete moments. These types of moments play an important role for measuring inequality, for example, income quantiles and Lorenz and Bonferroni curves. The $s$ th incomplete moment of $X$ is determined from (8) as

$$
m_{s}(y)=\int_{0}^{y} x^{s} f(x ; p, \alpha, \tau) d x=\sum_{j=0}^{\infty} w_{j} \int_{0}^{Q_{(j+1) \alpha, \tau}(y)} Q_{(j+1) \alpha, \tau}(u)^{s} d u .
$$

Both integrals in (13) can be evaluated numerically for most EGG distributions.

\section{Generating function}

An expression for the mgf of $X$ can be determined from Eq. (8) using the EG generating function. Initially, we can derive a simple linear combination for the mgf of $X$, say
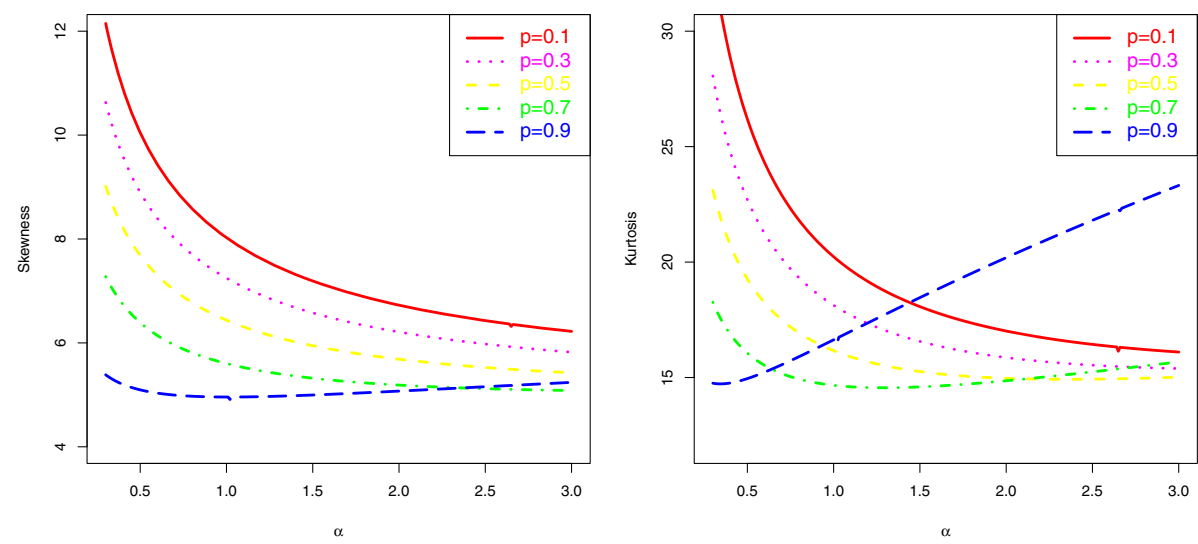

Fig. 3 Skewness and kurtosis of the XTGG distribution as functions of $\alpha$ for some values of $p$, with $\lambda=0.3$ and $\beta=0.8$ 

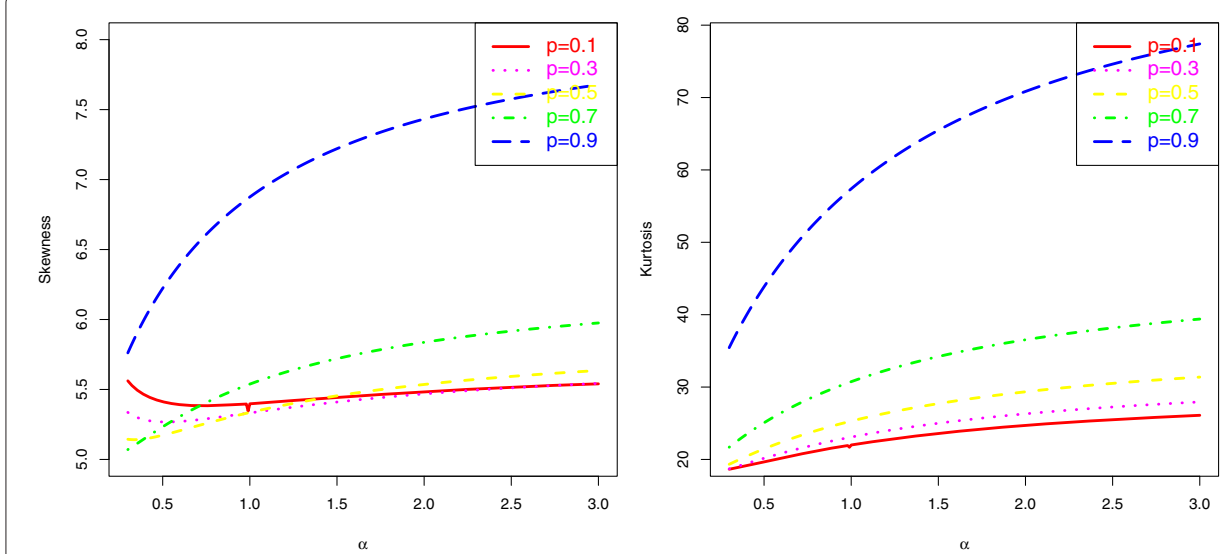

Fig. 4 Skewness and kurtosis of the MWG distribution as functions of $\alpha$ for some values of $p$, with $\lambda=0.04$ and $\gamma=0.8$

$M(t ; p, \alpha, \boldsymbol{\tau})$, from Eq. (8) given by

$$
M(t ; p, \alpha, \tau)=\sum_{j=0}^{\infty} w_{j} M_{(j+1) \alpha, \tau}(t),
$$

where $M_{\alpha, \tau}(t)=\int_{0}^{\infty} \mathrm{e}^{\mathrm{ty}} \mathrm{g}_{\alpha, \tau}(\mathrm{y})$ dy is the generating function of $Y_{\alpha}$. A simple formula for $M_{\alpha, \tau}(t)$ follows from Eqs. (2) and (3)

$$
M_{\alpha, \tau}(t)=\int_{0}^{1} \exp \left\{t Q_{\alpha, \tau}(u)\right\} d u
$$

Equations (14) and (15) are the main results of this section. Combining these two equations, we can derive the generating functions of some EGG special models.

\section{Mean deviations}

The amount of scatter in a population is measured to some extent by the totality of deviations from the mean and median. We can obtain the mean deviations about the ordinary mean $\mu_{1}^{\prime}=E(X)$ and about the median $M$ of $X$ using the relationships

$$
\delta_{1}=\int_{0}^{\infty}\left|t-\mu_{1}^{\prime}\right| f(t) d t \text { and } \delta_{2}=\int_{0}^{\infty}|t-M| f(t) d t
$$

and then

$$
\delta_{1}=2\left[\mu_{1}^{\prime} F\left(\mu_{1}^{\prime}\right)-m_{1}\left(\mu_{1}^{\prime}\right)\right] \text { and } \delta_{2}=\mu_{1}^{\prime}-2 m_{1}(M)
$$

where $\mu_{1}^{\prime}$ comes from (9) with $s=1$ and $M$ is given by $M=H^{-1}\left(\alpha^{-1} \log [(2-p)]\right)$. Further, we obtain from (13) with $s=1$

$$
m_{1}(y)=\sum_{j=0}^{\infty} w_{j} \int_{0}^{Q_{(j+1) \alpha, \tau}(y)} Q_{(j+1) \alpha, \tau}(u) d u .
$$


We define the quantity $A_{\alpha, \tau}(z)=\int_{0}^{z} y g_{\alpha, \tau}(y) d y=\int_{0}^{Q_{\alpha, \tau}(z)} Q_{\alpha, \tau}(u) d u$. A simple application of $A_{\alpha, \tau}(z)$ to the $\log$-Weibull (LW) distribution gives

$$
\begin{aligned}
A_{\alpha, \tau}(z) & =\mu G_{\alpha, \tau}(z)+\sigma \int_{0}^{G_{\alpha, \tau}(z)} \log \left\{-\frac{1}{\alpha} \log (1-u)\right\} d u \\
& =[\mu-\sigma \log (\alpha)] G_{\alpha, \tau}(z)+\sigma \int_{0}^{G_{\alpha, \tau}(z)} \log \{-\log (1-u)\} d u,
\end{aligned}
$$

where $G_{\alpha, \tau}(z)=1-\exp \left\{-\exp \left(\frac{t-\mu}{\sigma}\right)\right\}$.

Setting $v=1-u$, the integral can be determined using MAPLE as

$$
\int_{x}^{1} \log \{-\log (v)\} d v=-x \log [-\log (x)]-E_{i}(1,-\log (x))-\gamma,
$$

where $E_{i}(a, z)=\int_{1}^{\infty} x^{-a} \mathrm{e}^{\mathrm{zx}} \mathrm{dx}$ is the exponential integral and $\gamma$ is the Euler constant. Then,

$$
\begin{aligned}
A_{\alpha, \tau}(z)= & {[\mu-\sigma \log (\alpha)] G_{\alpha, \tau}(z)+\sigma\left\{-\left[1-G_{\alpha, \tau}(z)\right] \log \left\{-\log \left[1-G_{\alpha, \tau}(z)\right]\right\}\right.} \\
& \left.-E_{i}\left(1,-\log \left[1-G_{\alpha, \tau}(z)\right]\right)-\gamma\right\} .
\end{aligned}
$$

Finally, a practical application of $\delta_{1}$ and $\delta_{2}$ refers to the Bonferroni and Lorenz curves of $X$ defined by $B(\pi)=m_{1}(q) /\left(\pi \mu_{1}^{\prime}\right)$ and $L(\pi)=m_{1}(q) / \mu_{1}^{\prime}$, respectively, where $\pi$ is a specified probability and $q=F^{-1}(\pi)$ is given by

$$
q=H^{-1}\left(\alpha^{-1} \log \left[\frac{(1-p \pi)}{(1-\pi)}\right]\right) .
$$

These curves have applications in economics to study income and poverty, reliability, demography, insurance and medicine. Plots of the Bonferroni and Lorenz curves for the XTGG distribution for some parameter values are displayed in Fig. 5.

\section{Rényi entropy}

The entropy of a random variable $X$ is a measure of uncertainty variation. The Rényi entropy is defined by $I_{E G G}(\gamma)=\frac{1}{1-\gamma} \log \left\{\int_{\mathbb{R}} f^{\gamma}(x) d x\right\}$, where $\gamma>0$ and $\gamma \neq 1$. Based on the EGG density function (4) and using the power series (7), we obtain
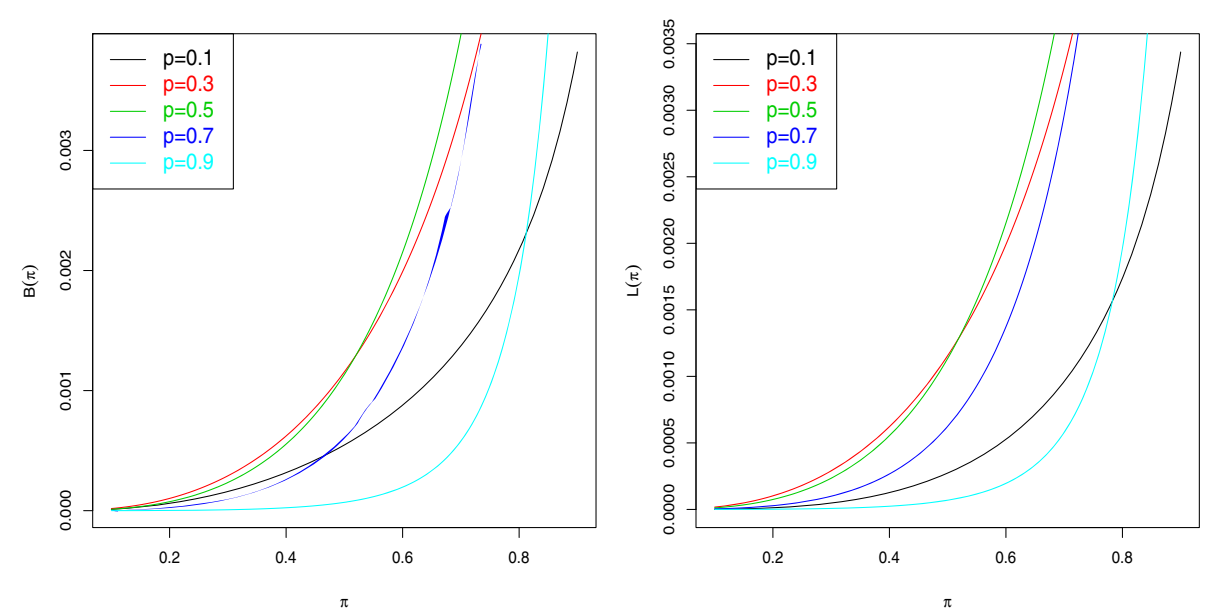

Fig. 5 Bonferroni and Lorenz curves for the XTGG distribution when $\lambda=0.3, \beta=0.8$ and $\alpha=2$ and some values of $p$ 


$$
f(x ; p, \alpha, \boldsymbol{\tau})^{\gamma}=\alpha^{\gamma}(1-p)^{\gamma} h(x)^{\gamma} \sum_{j=0}^{\infty} \frac{\Gamma(2 \gamma+j) p^{j}}{\Gamma(2 \gamma) j !} \exp \{-(\gamma+j) \alpha H(x)\}, \quad x>0 .
$$

Setting $\alpha_{j}^{\star}=(\gamma+j) \alpha / \gamma$, we can write $I_{E G G}(\gamma)$ as a linear combination of EG entropies. We obtain

$$
I_{E G G}(\gamma)=\frac{[\gamma(1-p)]^{\gamma}}{(1-\gamma)} \sum_{j=0}^{\infty} \frac{\Gamma(2 \gamma+j) p^{j}}{(\gamma+j)^{\gamma} \Gamma(2 \gamma) j !} I_{j}(\gamma)
$$

where $I_{j}(\gamma)$ denotes the EG Rényi entropy with scale parameter $\alpha_{j}^{\star}$. Hence, the EGG Rényi entropy is a linear combination of EG Rényi entropies with scale parameters given by $\alpha_{j}^{\star}$ for $j=0,1, \ldots$ The EG entropy depends on the form of $H(\cdot)$ and can be evaluated (at least numerically) using most statistical software programs.

\section{Order statistics}

Order statistics make their appearance in many areas of statistical theory and practice. The density function $f_{i: n}(x)$ of the $i$ th order statistic, say $X_{i: n}$, for $i=1, \ldots, n$, in a random sample of size $n$ from the EGG family can be expressed as

$$
f_{i: n}(x)=\frac{f(x)}{B(i, n-i+1)} \sum_{j=0}^{n-i}(-1)^{j}\left(\begin{array}{c}
n-i \\
j
\end{array}\right) F(x)^{i+j-1},
$$

where $B(\cdot, \cdot)$ is the beta function. Let $0<u=\exp \{-\alpha H(y)\}<1$. From Eqs. (4), (5) and (18), we can write

$$
f_{i: n}(x)=\frac{\alpha(1-p) h(x) u}{B(i, n-i+1)} \sum_{j=0}^{n-i}(-1)^{j}\left(\begin{array}{c}
n-i \\
j
\end{array}\right) \frac{(1-u)^{i+j-1}}{(1-p u)^{i+j+1}} .
$$

Using the binomial expansion and (7), we obtain after some algebra

$$
f_{i: n}(x)=\sum_{s=0}^{\infty} \sum_{j=0}^{n-i} \sum_{r=0}^{i+j-1} v_{i: n}(s, j, r) g_{\widetilde{\alpha}, \tau}(x)
$$

where $\widetilde{\alpha}=(r+s+1) \alpha$ and

$$
v_{i: n}(s, j, r)=(1-p) \sum_{s=0}^{\infty} \sum_{j=0}^{n-i} \sum_{r=0}^{i+j-1} \frac{(-1)^{j+r} n !(i+j+s) ! p^{s}}{(i+j)(r+s+1)(i-1) !(n-i-j) !(i+j-1-r) ! j ! r ! s !} .
$$

Equation (19) reveals that the density function of the EGG order statistics can be expressed as a triple linear mixture of EG densities. We have checked using MAPLE and MATHEMATICA that

$$
\sum_{s=0}^{\infty} \sum_{j=0}^{n-i} \sum_{r=0}^{i+j-1} v_{i: n}(s, j, r)=1 .
$$

The triple sums in (19) can be computed using most packages with a large number (say 20) of terms in place of infinity. So, we can derive several mathematical properties (ordinary and incomplete moments, mgf, etc) for the EGG order statistics directly from those quantities of the EG distribution. For example, the $p$ th ordinary moment of $X_{i: n}$ can be obtained from (19) and the quantity $\beta_{s}(\alpha, \tau)$ defined at the beginning of Section 3 as

$$
E\left(X_{i: n}^{p}\right)=\sum_{s=0}^{\infty} \sum_{j=0}^{n-i} \sum_{r=0}^{i+j-1} v_{i: n}(s, j, r) \beta_{p}(\widetilde{\alpha}, \boldsymbol{\tau}) .
$$




\section{Maximum likelihood estimation}

We determine the maximum likelihood estimates (MLEs) of the parameters of the EGG family from complete samples only. Let $x_{1}, \ldots, x_{n}$ be a random sample of size $n$ from the EGG $(p, \alpha, \tau)$ distribution. The log-likelihood function for the vector of parameters $\boldsymbol{\theta}=(p, \alpha, \boldsymbol{\tau})^{T}$ becomes

$$
\begin{aligned}
l(\boldsymbol{\theta}) & =n[\log (\alpha)+\log (1-p)]+\sum_{i=1}^{n} \log \left[h\left(x_{i}\right)\right]-\alpha \sum_{i=1}^{n} H\left(x_{i}\right) \\
& -2 \sum_{i=1}^{n} \log \left[1-p \exp \left\{-\alpha H\left(x_{i}\right)\right\}\right] .
\end{aligned}
$$

The components of the score vector $U(\boldsymbol{\theta})$ are obtained by differentiating (21)

$$
\begin{aligned}
& U_{p}(\boldsymbol{\theta})=-\frac{n}{(1-p)}+2 \sum_{i=1}^{n} \frac{\exp \left\{-\alpha H\left(x_{i}\right)\right\}}{1-p \exp \left\{-\alpha H\left(x_{i}\right)\right\}}, \\
& U_{\alpha}(\boldsymbol{\theta})=\frac{n}{\alpha}-\sum_{i=1}^{n} H\left(x_{i}\right)+2 \sum_{i=1}^{n} \frac{p H\left(x_{i}\right) \exp \left\{-\alpha H\left(x_{i}\right)\right\}}{1-p \exp \left\{-\alpha H\left(x_{i}\right)\right\}} \\
& U_{\boldsymbol{\tau}}(\boldsymbol{\theta})=\sum_{i=1}^{n} \frac{\dot{h}\left(x_{i}\right)_{\tau}}{h\left(x_{i}\right)}-\alpha \sum_{i=1}^{n} \dot{H}\left(x_{i}\right)_{\tau}-2 \sum_{i=1}^{n} \frac{\alpha p \dot{H}\left(x_{i}\right)_{\tau} \exp \left\{-\alpha H\left(x_{i}\right)\right\}}{1-p \exp \left\{-\alpha H\left(x_{i}\right)\right\}},
\end{aligned}
$$

where $\dot{h}\left(x_{i}\right)_{\tau}=\partial h\left(x_{i}\right) / \partial \tau$ and $\dot{H}\left(x_{i}\right)_{\tau}=\partial H\left(x_{i}\right) / \partial \tau$ are $p \times 1$ vectors. Because the equations $U_{p}(\boldsymbol{\theta})=0, U_{\alpha}(\boldsymbol{\theta})=0$ and $U_{\boldsymbol{\tau}}(\boldsymbol{\theta})=0$ are nonlinear, the MLE $\widehat{\boldsymbol{\theta}}$ have to be evaluated numerically. The log-likelihood can be maximized either directly by using the MaxBFGS routine in the matrix programming language Ox (see, Doornik 2007).

For interval estimation and hypothesis tests on the model parameters, we require the observed information matrix $J=J(\theta)$, whose elements can be evaluated numerically. Under general regularity conditions, the asymptotic distribution of $(\widehat{\theta}-$ $\boldsymbol{\theta})$ is $N_{p+2}\left(0, I(\boldsymbol{\theta})^{-1}\right)$, where $I(\boldsymbol{\theta})$ is the expected information matrix. We can substitute $I(\boldsymbol{\theta})$ by the observed information matrix $J(\widehat{\boldsymbol{\theta}})$ evaluated at $\widehat{\boldsymbol{\theta}}$. The multivariate normal $N_{p+2}\left(0, J(\widehat{\boldsymbol{\theta}})^{-1}\right)$ distribution can be used to obtain approximate confidence intervals for the individual parameters.

We can compute the maximum values of the unrestricted and restricted log-likelihoods to construct likelihood ratio (LR) statistics for testing some sub-models of the EGG family. For example, we may use the LR statistic to check if the fit using the MWG distribution is statistically "superior" to a fit using the MW distribution for a given data set. In any case, hypothesis tests of the type $H_{0}: \boldsymbol{\theta}=\boldsymbol{\theta}_{0}$ versus $H: \boldsymbol{\theta} \neq \boldsymbol{\theta}_{0}$, can be performed using LR statistics. For example, the comparison of the MWG and MW distributions, where $\boldsymbol{\theta}=(p, \alpha, \lambda, \gamma)^{T}$, is equivalent to test $H_{0}: p=0$ versus $H: H_{0}$ is not true. The LR statistic reduces to

$$
w=2\{\ell(\widehat{p}, 1, \widehat{\alpha}, \widehat{\lambda}, \widehat{\gamma})-\ell(0, \widetilde{\alpha}, \widetilde{\lambda}, \widetilde{\gamma})\},
$$

where $\widehat{p}, \widehat{\alpha}, \widehat{\lambda}$ and $\widehat{\gamma}$ are the MLEs under $H$ and $\widetilde{\alpha}, \tilde{\lambda}$ and $\tilde{\gamma}$ are the estimates under $H_{0}$.

We apply formal goodness-of-fit tests in order to verify which distribution gives the best fit. We consider the Cramér-Von Mises $\left(W^{*}\right)$ and Anderson-Darling $\left(A^{*}\right)$ statistics. In general, the smaller the values of these statistics, the better the fit to the data. Let $F(x ; \boldsymbol{\theta})$ be the cdf, where the form of $F$ is known but $\boldsymbol{\theta}$ (a $k$-dimensional parameter vector, say) 
is unknown. To obtain the $W^{*}$ and $A^{*}$ statistics, we can proceed as follows: (i) compute $v_{i}=F\left(x_{i} ; \widehat{\boldsymbol{\theta}}\right)$, where the $x_{i}$ 's are in ascending order, $y_{i}=\Phi^{-1}\left(v_{i}\right)$ is the standard normal qf and $u_{i}=\Phi\left\{\left(y_{i}-\bar{y}\right) / s_{y}\right\}$, where $\bar{y}=n^{-1} \sum_{i=1}^{n} y_{i}$ and $s_{y}^{2}=(n-1)^{-1} \sum_{i=1}^{n}\left(y_{i}-\bar{y}\right)^{2}$; (ii) calculate $W^{2}=\sum_{i=1}^{n}\left\{u_{i}-(2 i-1) /(2 n)\right\}^{2}+1 /(12 n)$ and $A^{2}=-n-n^{-1} \sum_{i=1}^{n}\{(2 i-$ 1) $\left.\log \left(u_{i}\right)+(2 n+1-2 i) \log \left(1-u_{i}\right)\right\}$ and (iii) modify $W^{2}$ into $W^{*}=W^{2}(1+0.5 / n)$ and $A^{*}$ into $A^{*}=A^{2}\left(1+0.75 / n+2.25 / n^{2}\right)$. For further details, the reader is referred to Chen and Balakrishnan (1995).

\section{Application: ozone level data}

We illustrate the superiority of some EGG distributions over their sub-models. We consider the data set from the New York State Department of Conservation corresponding to the daily ozone level measurements in New York in May-September, 1973. The numerical evaluations are performed using the MaxBFGS sub-routine of the Ox program (Doornik 2007).

We consider the reparametrizations, $\alpha=\alpha_{1}^{-\gamma}$ for the MWG model, $\alpha=\alpha_{1}^{-\beta}$ for the XTGG model and $\alpha_{1}=\alpha_{3}^{-\beta_{1}}$ and $\alpha_{2}=\alpha_{4}^{-\beta_{2}}$ for the AWG model. The density functions of the XTGG and MWG models are presented in the "Appendix".

Alternative models to fit these data can be based on the Burr XII (BXII) distribution. There are various extensions of this distribution. Recently, Lanjoni et al. (2015) introduced two extended BXII distributions by compounding the BXII and geometric distributions. These models are called the BXII geometric type I (BXIIGI) and BXII geometric type II (BXIIGII) distributions, whose density functions are given below:

\section{- BXIIGI distribution}

$$
f(x ; s, k, c, p)=\frac{(1-p) c k s^{-c} x^{c-1}\left[1+\left(\frac{x}{s}\right)^{c}\right]^{-k-1}}{\left\{1-p\left[1+\left(\frac{x}{s}\right)^{c}\right]^{-k}\right\}^{2}}, \quad x>0,
$$

where $s>0$ is a scale parameter and $k>0, c>0$ and $p \in(0,1)$ are shape parameters.

\section{- BXIIGII distribution}

$$
f(x ; s, k, c, p)=\frac{(1-p) c k s^{-c} x^{c-1}\left[1+\left(\frac{x}{s}\right)^{c}\right]^{-k-1}}{\left\{1-p+p\left[1+\left(\frac{x}{s}\right)^{c}\right]^{-k}\right\}^{2}} \quad x>0 .
$$

We fit the MWG, XTGG and AWG models, their parent distributions and the BXIIGI and BXIIGII models to the current data. Table 2 lists the MLEs of the model parameters, their standard errors (in parentheses) and the values of the Akaike information criterion (AIC). These results indicate that the special MW, XTG and AW models have larger AIC values when compared with those values of the new MWG, XTGG and AWG models. Since the value of the AIC statistic is smaller for the XTGG distribution compared to the values of the other five models, this distribution can be chosen as the best model to fit these data among these models.

The values of the $W^{*}$ and $A^{*}$ statistics for all eight fitted models are given in Table 3 . Thus, according to these statistics, the MWG, XTGG and AWG models fit the current data better than their sub-models. The figures in this table also reveal that the XTGG model has the lowest values for the $W^{*}$ and $A^{*}$ statistics among all eight fitted models. Hence, it could be chosen as the best model to explain these data. Figure 6 displays the histogram of the data and some plots comparing the fitted densities. We can also conclude 
Table 2 MLEs of the model parameters for the daily ozone level data, the corresponding SEs (given in parentheses) and the AIC statistics

\begin{tabular}{|c|c|c|c|c|c|c|}
\hline Model & $p$ & $\alpha_{1}$ & $\lambda$ & $\gamma$ & & AIC \\
\hline \multirow[t]{2}{*}{$\overline{M W G}$} & 0.83032 & 104.81 & 0.0030932 & 1.6764 & & 1089.21 \\
\hline & $(0.18959)$ & (78.852) & $(0.0068905)$ & $(0.23498)$ & & \\
\hline \multirow[t]{3}{*}{ MW } & - & 46.080 & $2.9046 \mathrm{e}-013$ & 1.3402 & & 1091.22 \\
\hline & & (3.3755) & $(0.00053284)$ & ( 0.095420$)$ & & \\
\hline & $p$ & $\alpha_{1}$ & $\lambda$ & $\beta$ & & AIC \\
\hline \multirow[t]{2}{*}{ XTGG } & 0.92198 & 19.384 & 119.79 & 1.7396 & - & 1088.39 \\
\hline & $(0.16726)$ & (28.978) & $(92.031)$ & (0.19738) & & \\
\hline \multirow[t]{3}{*}{ XTG } & - & 3.7020 & 20682. & 1.3401 & - & 1091.22 \\
\hline & & $(1.8864)$ & (85.071) & $(0.095512)$ & & \\
\hline & $p$ & $\alpha_{3}$ & $\beta_{1}$ & $\alpha_{4}$ & $\beta_{2}$ & AIC \\
\hline \multirow[t]{2}{*}{ AWG } & 0.75545 & 0.99528 & 116.54 & 0.99528 & 116.54 & 1091.45 \\
\hline & $(0.17410)$ & (0.0393) & (982.7309) & (0.0393) & (0.0393) & \\
\hline \multirow[t]{3}{*}{ AW } & - & 0.99622 & 77.29 & 0.99622 & 77.29 & 1093.22 \\
\hline & & $(0.0251070)$ & (512.43) & $(0.025107)$ & $(512.43)$ & \\
\hline & $p$ & s & $c$ & k & & AIC \\
\hline \multirow[t]{2}{*}{$B X \| G \mid$} & 0.7553 & 16102 & 1.7363 & 10405 & - & 1089.5 \\
\hline & $(0.1760)$ & (4316.05) & $(0.2089)$ & (3936.62) & & \\
\hline \multirow[t]{2}{*}{$B X\|G\|$} & $1 \mathrm{E}-8$ & 134.00 & 1.4860 & 5.5678 & - & 1092.7 \\
\hline & $(1 \mathrm{E}-9)$ & $(17.35)$ & $(0.2235)$ & $(2.4753)$ & & \\
\hline
\end{tabular}

that the distributions in the new family are very competitive models for describing the daily ozone level data. Finally, these results prove empirically the potentiality of the EGG family.

\section{Concluding remarks}

In this paper, we introduce and study a new class of distributions called the extendedG geometric (EGG) family that generalizes the Weibull geometric and modified Weibull geometric distributions proposed by Barreto-Souza et al. (2011) and Wang and Elbatal (2015), respectively, among other distributions. This is achieved by compounding the class of extended-G (EG) distributions (Nadarajah and Kotz 2005) with the geometric distribution. The EGG family is quite flexible in analyzing positive data instead of some other special models.

Table 3 Formal tests for some models

\begin{tabular}{ccc}
\hline Model & $W^{*}$ & Statistics \\
\hline MWG & 0.091 & $A^{*}$ \\
MW & 0.170 & 0.545 \\
XTGG & 0.078 & 0.966 \\
XTG & 0.170 & 0.474 \\
AWG & 0.088 & 0.966 \\
AW & 0.170 & 0.539 \\
BXIIGI & 0.088 & 0.966 \\
BXIIGII & 0.139 & 0.539 \\
\hline
\end{tabular}




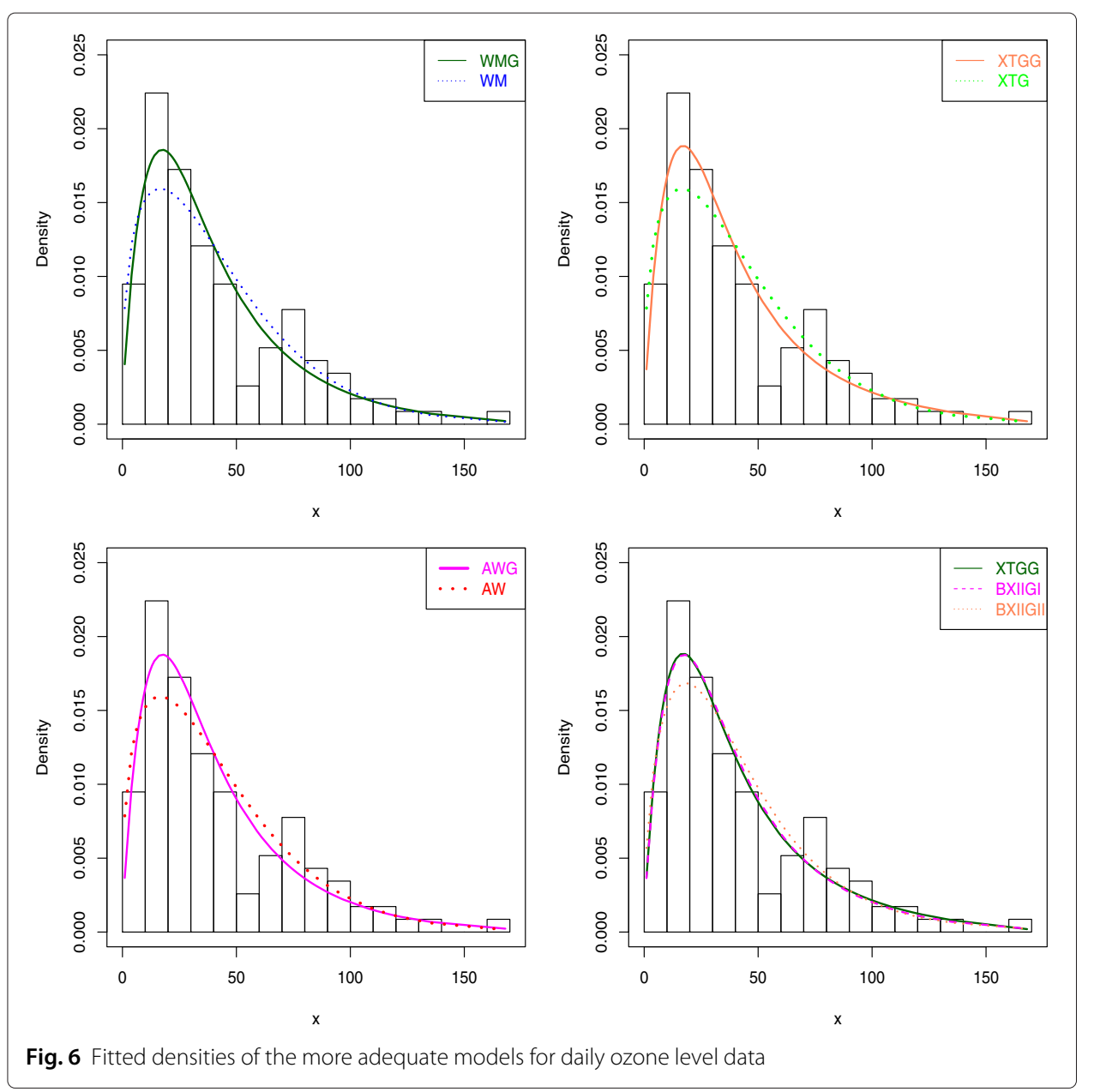

The density function of the EGG family can be expressed as a linear mixture of EG densities. We provide a mathematical treatment of the new family including explicit expressions for the ordinary and incomplete moments, generating function, mean deviations, Bonferroni and Lorenz curves, order statistics and their moments. The estimation of the parameters is approached by the method of maximum likelihood. An application to real lifetime data indicate that the EGG family could provide better fits than other well-known lifetime models. We expect that the new family of models will attract wider applications in Statistics.

\section{Appendix: new special EGG models}

\section{- Modified Weibull geometric distribution}

The case $H(x)=x^{\gamma} \exp (\lambda x)$ and $h(x)=x^{\gamma-1} \exp (\lambda x)(\gamma+\lambda x)$, where $\gamma>0$ and $\lambda \geq 0$, in Eq. (4), gives the modified Weibull geometric (MWG) distribution. If $p \rightarrow 0^{+}$, it reduces to the MW distribution. The MWG distribution is very flexible to accommodate a hrf that has increasing, decreasing, bathtub and unimodal shapes. It is also suitable for testing goodness of fit of some special models such as the modified Weibull (MW), Weibull geometric (WG) and Weibull distributions.

\section{- Exponential power geometric distribution}


For the case $H(x)=\exp \left[(\lambda x)^{\beta}\right]-1, h(x)=\beta \lambda \exp \left[(\lambda x)^{\beta}\right](\lambda x)^{\beta-1}$ and $\alpha=1$, where $\beta, \lambda>0$, we obtain the exponential power geometric (EPG) distribution. If $p \rightarrow 0^{+}$in addition to $\alpha=1$, it becomes the exponential power (EP) distribution (Smith and Bain 1975). This distribution has the property that its hrf may take a U-shaped form. Smith and Bain (1975) presented some general properties of least squares estimators and discussed the case of location-scale parameter distributions.

\section{- Chen geometric distribution}

The case $H(x)=\exp \left(x^{\beta}\right)-1, h(x)=\beta x^{\beta-1} \exp \left(x^{\beta}\right)$, where $\beta>0$, corresponds to the Chen geometric (CG) distribution. If $p \rightarrow 0^{+}$, it becomes the Chen distribution (Chen, 2000), which has increasing or bathtub-shaped hrf. Chen (2000) discussed exact confidence intervals and exact joint confidence regions for the parameters based on type-II censored samples.

\section{- XTG geometric distribution}

For $H(x)=\lambda\left[\exp \left\{(x / \lambda)^{\beta}\right\}-1\right]$ and $h(x)=\beta \exp \left\{(x / \lambda)^{\beta}\right\}(x / \lambda)^{\beta-1}$, where $\beta>0$ and $\lambda>0$, we obtain the XTG geometric (XTGG) distribution. If $p \rightarrow 0^{+}$, it reduces to Xie et al.'s (2002) model. They studied parameter estimation methods and showed its applicability. If $\lambda=1$, this model reduces to the CG distribution above.

\section{- Log-Weibull geometric distribution}

For $H(x)=\exp [(x-\mu) / \sigma], h(x)=(1 / \sigma) \exp [(x-\mu) / \sigma]$ and $\alpha=1$, where $-\infty<\mu<\infty$ and $\sigma>0$, we have the log-Weibull geometric (LWG) distribution. If $p \rightarrow 0^{+}$, it gives as special case the log-Weibull (LW) distribution (White 1969; Lawless 2003). White (1969) obtained the means and variances of the order statistics of the LW distribution and listed these values in special tables. Examples of these tables to obtain weighted least squares estimates from censored samples from a Weibull distribution are also presented. The LW distribution is a very popular distribution for modeling lifetime data and phenomenon with monotone failure rates.

\section{- Kies geometric distribution}

For $H(x)=[(x-\mu) /(\sigma-x)]^{\beta}$ and $h(x)=\beta[(x-\mu) /(\sigma-x)]^{\beta-1}\left[(\sigma-\mu) /(\sigma-x)^{2}\right]$, where $0<\mu<t<\sigma<\infty$, we obtain the Kies geometric (KG) distribution. If $p \rightarrow 0^{+}$, it yields as a special model the Kies distribution (Kies 1958), which is an extension of the Weibull distribution for strength modeling.

\section{- Phani geometric distribution}

The case $H(x)=(x-\mu)^{\beta_{1}} /(\sigma-x)^{\beta_{2}}$ and $h(x)=(x-\mu)^{\beta_{1}-1}(\sigma-x)^{-\left(\beta_{2}-1\right)}$ $\left[\beta_{1}(\sigma-x)+\beta_{2}(x-\mu)\right]$, where $0<\mu<t<\sigma<\infty$ and $\beta_{1}, \beta_{2}>0$, leads to the Phani geometric (PG) distribution. If $p \rightarrow 0^{+}$, it reduces to the Phani distribution (Phani 1987). If $\beta_{1}=\beta_{2}=\beta$, it gives the KG distribution discussed before. Phani (1987) presented statistical justification for modifying the Weibull distribution for the analysis of fibre strength data.

\section{- Additive Weibull geometric distribution}

For $H(x)=\left(x / \beta_{1}\right)^{\alpha_{1}}+\left(x / \beta_{2}\right)^{\alpha_{2}}, h(x)=\left(\alpha_{1} / \beta_{1}\right)\left(x / \beta_{1}\right)^{\alpha_{1}-1}+\left(\alpha_{2} / \beta_{2}\right)\left(x / \beta_{2}\right)^{\alpha_{2}-1}$ and $\alpha=1$, where $\alpha_{1}, \alpha_{2}, \beta_{1}, \beta_{2}>0$, we obtain the additive Weibull geometric (AWG) distribution. If $a=b=1$ in addition to $\alpha=1$, it gives the additive Weibull (AW) distribution (Xie and Lai 1995). This distribution has a bathtub-shaped failure rate function. Xie and Lai (1995) studied a simple model based on adding two Weibull survival functions, presented some simplifications of the model and analyzed the graphical estimation technique based on the conventional Weibull plot. 


\section{- Generalized power Weibull geometric distribution}

For $H(x)=\left[1+(x / \beta)^{\alpha_{1}}\right]^{\theta}-1, h(x)=\left(\theta \alpha_{1} / \beta\right)\left[1+(x / \beta)^{\alpha_{1}}\right]^{\theta-1}(x / \beta)^{\alpha_{1}}$ and $\alpha=1$, where $\alpha_{1}, \beta>0$ and $\theta \geq 0$, we obtain the generalized power Weibull geometric (GPWG) distribution. If $p \rightarrow 0^{+}$, it becomes the generalized power Weibull (GPW) distribution (Nikulin and Haghighi 2006). They proposed a chi-squared type statistic to test the validity of the GPW family based on the head-and-neck cancer censored data.

\section{- BLZ geometric distribution}

The case $H(x)=\exp \left(\alpha_{1} x-\beta / x\right), h(x)=\exp \left(\alpha_{1} x-\beta / x\right)\left(\alpha_{1}+\beta x^{-2}\right)$ and $\alpha=1$, where $\alpha_{1}, \beta>0$, leads to the BLZ geometric (BLZG) distribution. It extends the Bebbington et al.'s (2007) distribution, which corresponds to $p \rightarrow 0^{+}$and $\alpha=1$. Its hrf has four different forms: bathtub shape, increasing, decreasing and upside-down bathtub. They presented the BLZ distribution as an extension of the Weibull, studied its properties and derived explicit formulas for the turning points of the failure rate function in terms of its parameters.

\section{- Gompertz geometric distribution}

For $H(x)=\alpha_{1}^{-1}\left[\exp \left(\alpha_{1} x\right)-1\right]$ and $h(x)=\exp \left(\alpha_{1} x\right)$, where $-\infty<\alpha_{1}<\infty$, we obtain the Gompertz geometric (GG) distribution. If $p \rightarrow 0^{+}$, it gives the Gompertz distribution (Pham and Lai 2007).

\section{- Pham geometric distribution}

For $H(x)=a_{1}^{x_{1}^{\alpha_{1}}}-1, h(x)=\alpha_{1} x^{\alpha_{1}-1} \log \left(a_{1}\right) a_{1}^{x^{\alpha_{1}}}$ and $\alpha=1$, where $\alpha_{1}, a_{1}>0$, we obtain the Pham geometric (PG) distribution. If $p \rightarrow 0^{+}$, it yields the Pham distribution (Pham 2002). Pham (2002) proposed this distribution, also referred to as the log-log distribution, and showed that it has a U-shaped hrf.

\section{- Nadarajah-Kotz geometric distribution}

For $H(x)=x^{b_{1}}\left[\exp \left(c x^{d}\right)-1\right]$ and $h(x)=b_{1} x^{b_{1}-1}\left[\exp \left(c x^{d}\right)-1\right]+c d x^{\left(b_{1}+d-1\right)} \exp \left(c x^{d}\right)$, where $b_{1}, c \geq 0$ and $d>0$, we obtain the Nadarajah-Kotz geometric (NKG) distribution. If $p \rightarrow 0^{+}$, it gives the distribution due to Nadarajah and Kotz (2005). They presented some modifications of the Weibull distribution and also discussed some modifications suggested by Gurvich et al. (1997). For $b_{1}=0$ and $c=1$, this model reduces to the CG distribution discussed before.

\section{- Slymen-Lachenbruch geometric distribution}

$$
\text { For } H(x)=\exp \left[\alpha_{1}+(\beta / 2 \theta)\left(x^{\theta}-x^{-\theta}\right)\right] \text {, }
$$

$h(x)=(\beta / 2 x) \exp \left[\alpha_{1}+(\beta / 2 \theta)\left(x^{\theta}-x^{-\theta}\right)\right]\left(x^{\theta}+x^{-\theta}\right)$ and $\alpha=1$, where $\alpha_{1}, \theta>0$, we obtain the Slymen-Lachenbruch geometric (SLG) distribution. If $p \rightarrow 0^{+}$, it becomes the distribution proposed by Slymen and Lachenbruch (1984). They introduced and studied two classes of distributions within the framework of parametric survival analysis. These classes are derived from a general linear form by specifying a function of the survival function under certain restrictions.

We now give the density functions for two distributions in the EGG family.

1. The XTGG density function is given by

$$
\begin{aligned}
f(x)= & \alpha \beta \exp \left\{(x / \lambda)^{\beta}\right\}(x / \lambda)^{\beta-1} \exp \left\{-\alpha \lambda\left[\exp \left\{(x / \lambda)^{\beta}\right\}-1\right]\right\}(1-p) \\
& {\left[1-p \exp \left\{-\alpha \lambda\left[\exp \left\{(x / \lambda)^{\beta}\right\}-1\right]\right\}\right]^{-2} . }
\end{aligned}
$$


If $X$ is a random variable with density function (24), we write $X \sim \operatorname{XTGG}(p, \alpha, \lambda, \beta)$.

2. The MWG density function is given by

$$
f(x)=\frac{\alpha x^{\gamma-1} \exp (\lambda x)(\gamma+\lambda x) \exp \left\{-\alpha x^{\gamma} \exp (\lambda x)\right\}(1-p)^{2}}{\left\{1-p\left[\exp \left\{-\alpha x^{\gamma-1} \exp (\lambda x)\right\}\right]\right\}} .
$$

If $X$ is a random variable with density function (25), we write $X \sim \operatorname{MWG}(p, \alpha, \lambda, \gamma)$.

Acknowledgments

The financial support from CNPq is gratefully acknowledged.

\section{Author details}

${ }_{1}^{1}$ Departamento de Estatística, Universidade Federal de Pernambuco, Cidade Universitária, 50740-540 Recife, PE, Brazil. ${ }^{2}$ Departamento de Estatística, Universidade Federal da Bahia, Av. Adhemar de Barros, s/nº, 40170-110 Salvador, Brazil.

${ }^{3}$ Departamento de Ciências Exatas, Universidade de São Paulo, Av. Pádua Dias 11, 13418-900 Piracicaba, SP, Brazil.

Received: 31 July 2015 Accepted: 3 February 2016

Published online: 16 February 2016

\section{References}

Adamidis, K, Loukas, S: A Lifetime distribution with decreasing failure rate. Stat. Probab. Lett. 39, 35-42 (1998)

Barreto-Souza, W, de Morais, AL, Cordeiro, GM: The Weibull-geometric distribution. J. Stat. Comput. Simul. 81, 645-657 (2011)

Bebbington, M, Lai, CD, Zitikis, R: A flexible Weibull extension. Reliab. Eng. Syst. Saf. 92, 719-726 (2007)

Carrasco, JMF, Ortega, EMM, Cordeiro, GM: A generalized modified Weibull distribution for lifetime modeling. Comput. Stat. Data Anal. 53, 450-462 (2008)

Chen, G, Balakrishnan, N: A general purpose approximate goodness-of-fit test. J. Qual. Technol. 27, 154-161 (1995)

Chen, ZA: A new two-parameter lifetime distribution with bathtub shape or increasing failure rate function. Stat. Probab. Lett. 49, 155-61 (2000)

Cordeiro, GM, Ortega, EMM, Silva, GO: The Beta Weibull geometric distribution. Statistics. 47, 817-834 (2013)

de Santana, TVF, Ortega, EMM, Cordeiro, GM, Silva, GO: The Kumaraswamy log-logistic distribution. J. Stat. Theory Appl. 11, 265-291 (2012)

Doornik, JA: An Object-Oriented Matrix Language Ox 5. Timberlake Consultants Press, London (2007)

Gupta, RD, Kundu, D: Generalized exponential distribution. Australian and New Zealand J. Stat. 41, 173-188 (1999)

Gupta, RD, Kundu, D: Exponetiated exponential family: an alternative to gamma and Weibull distributions. Biom. J. 43, $117-130$ (2001a)

Gupta, RD, Kundu, D: Generalized exponential distribution: Different method of estimations. J. Stat. Comput. Simul. 69, $315-337$ (2001b)

Gurvich, MR, Dibenedetto, AT, Ranade, SV: A new statistical distribution for characterizing the random strength of brittle materials. J. Mater. Sci. 32, 2559-2564 (1997)

Kies, JA: Naval Research Laboratory (U.S.) and Naval Postgraduate School (U.S.): The Strength of Glass. NRL report. Naval Research Laboratory (1958). https://books.google.com.br/books?id=wVnYSAAACAAJ

Lanjoni, EM, Ortega, EMM, Cordeiro, GM: Extended Burr XII regression models: theory and applications. J. Agric. Biol. Environ. Stat (2015). doi:10.1007/s13253-015-0236-z

Lai, CD, Xie, M, Murthy, DNP: A modified Weibull distribution. Trans. Reliab. 52, 33-37 (2003)

Lawless, JF: Statistical Models and Methods for Lifetime Data (2003)

Mudholkar, GS, Srivastava, DK, Friemer, M: The exponentiated Weibull family: A reanalysis of the bus-motor-failure data. Technometrics. 37, 436-445 (1995)

Mudholkar, GS, Srivastava, DK, Kollia, GD: A generalization of the Weibull distribution with application to the analysis of survival data. J. Am. Stat. Assoc. 91, 1575-1583 (1996)

Nadarajah, S, Kotz, S: On some recent modifications of Weibull distribution. IEEE Trans. Reliab. 54, 561-562 (2005)

Nadarajah, S, Kotz, S: The beta exponential distribution. Reliab. Eng. Syst. Saf. 91, 689-697 (2006)

Nikulin, M, Haghighi, F: A Chi-squared test for the generalized power Weibull family for the head-and-neck cancer censored data. J. Math. Sci. 133, 1333-1341 (2006)

Pham, H: A bathtub-shaped hazard rate function with applications to system safety. Int. J. Reliabil. Appl. 3, 1-16 (2002)

Pham, H, Lai, CD: On recent generalizations of the Weibull distribution. IEEE Trans. Reliab. 56, 454-458 (2007)

Phani, KK: A new modified Weibull distribution. Commun. Am. Ceramic Soc. 70, 182-184 (1987)

Prudnikov, AP, Brychkov, YA, Marichev, OI: Integrals and Series, volume 1 (1986)

Silva, GO, Ortega, EMM, Cordeiro, GM: The Beta modified Weibull distribution. Lifetime Data Anal. 16, 409-430 (2010a)

Silva, RB, Barreto-Souza, W, Cordeiro, GM: A new distribution with decreasing, increasing and upside-down bathtub failure rate. Comput. Stat. Data Anal. 54, 935-944 (2010b)

Slymen, DJ, Lachenbruch, PA: Survival distributions arising from two families and generated by transformations. Commun. Stat. Theory Methods. 13, 1179-1201 (1984)

Smith, RM, Bain, LJ: An exponential power life testing distributions. Commun. Stat. 4, 469-481 (1975)

Xie, M, Lai, CD: Reliability analysis using an additive Weibull model with bathtub-shaped failure rate function. Reliab. Eng. Syst. Saf. 52, 87-93 (1995)

Xie, M, Tang, Y, Goh, TN: A modified Weibull extension with bathtub failure rate function. Reliab. Eng. Syst. Saf. 76 279-285 (2002)

Wang, M, Elbatal, I: The modified Weibull geometric distribution. Metron. 73, 303-315 (2015)

White, JS: The moments of log-Weibull order statistics. Technometrics. 11, 373-386 (1969) 